\title{
STUDY OF SOOT PARAMETERS IN THE PROCESS OF COMBUSTION OF CONDENSED SYSTEMS
}

\author{
Vladimir V. Promahov ${ }^{1, *}$, Tatyana V. Zhukova ${ }^{1}$, and Yuliya N. Ryzhikh ${ }^{1}$ \\ ${ }^{1}$ National Research Tomsk State University, 634050 Tomsk, Russia
}

\begin{abstract}
In the present information presents the results of studies of the effect of dispersion of ammonium perchlorate on the content and nature of the soot formed in the process of burning of butyl-rubber propellants.
\end{abstract}

Combustion products of condensed substances based on ammonium perchlorate (AP) contain soot-like substances. The laws of formation of these substances and their characteristics are not described in literature.

Results of study of influence of dispersity of ammonium perchlorate on the nature and content of the soot formed in the process of burning of butyl-rubber propellants (oxidizer excess ratio $\approx 0.43$ ) with the following composition:

ammonium perchlorate $-73 \%$, butyl rubber $-24.8 \%$, quinol ether $-2.2 \%$.

Oxidizer dispersity was changed during the tests. AP of three types: fine fraction (less than $50 \mu$ ), coarse fraction $(200 \div 300) \mu$ and bidisperse (mixture of the two previous fractions in the ratio of $1: 1)$ was used. Samples were burned in the pressure range of $(1 \div 8)$ $\mathrm{MPa}$ in nitrogen atmosphere.

Soot content was measured using two methods - laser diagnostics and direct collection of soot formed after burning up of a sample in a constant-pressure device.

A new approach was used for optical diagnostics. This approach makes it possible to determine both mass concentration and maximum size of condensed particles on the basis of the data of laser probing of combustion products.

According to offered method measurements of optical density $\lambda^{\text {meas }}(\lambda)$ of a dispersed medium are performed in several equally-spaced points of spectral wavelength range $\lambda=\lambda_{\min } \div \lambda_{\text {max }}$. The boundaries of this range are selected on the basis of valid information about complex refractive index $m=n-\mathrm{i} \cdot æ$.

Particle mass concentration is determined for the range $\lambda \geq \lambda_{*}$; maximum particle size is determined using boundary wavelength $\lambda_{*}$ and associated parameter $\alpha_{*}$ according to the following relations:

$$
C_{m}=\frac{\tau^{\text {meas }}(\lambda)}{C \cdot K^{(p)}(\lambda)},
$$

\footnotetext{
* Corresponding author: bvborisov@tpu.ru
} 


$$
D_{m}=\frac{\alpha_{*} \lambda_{*}}{\pi}
$$

where $C=$ const $=\frac{1.5 l}{\rho_{k}}, K^{(p)}(\lambda)-$ coefficient in Rayleigh formula for attenuation efficiency factor

$Q(\alpha, m), \alpha_{*}=f\left(\lambda_{*}\right), l-$ flow diameter, $\rho_{k}$ - particle material density.

Boundary value $\lambda_{*}$ (fig.1) is determined as abscissa of inflection point of the following function

$$
\bar{\tau}(\lambda)=\frac{\tau^{\text {meas }}(\lambda)}{K^{(p)}(\lambda)} .
$$

Expression for optical density $\tau(\lambda)$ for monodisperse particles in the framework of assumptions of Mie theory has the following form [1]:

$$
\tau(\lambda)=\frac{1.5 C_{m} l}{\rho_{k} D} Q(\alpha, m) .
$$

Dependences $n(\lambda)$, $(\lambda)$ have a complex character and are determined experimentally.

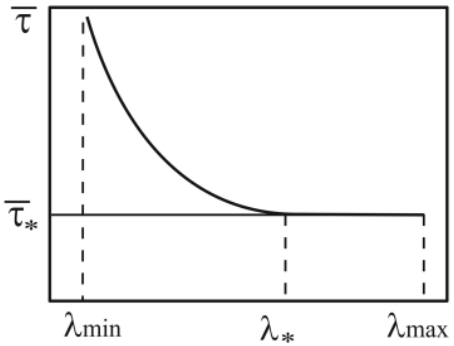

Fig. 1. Characteristic values of probe radiation wavelength.

Attenuation efficiency factor for $D<<\lambda$ (Rayleigh approximation) is determined using the formula [1]:

$$
Q^{(P)}(\lambda)=\frac{24 n æ}{\left(n^{2}-\mathfrak{x}^{2}+2\right)^{2}+(2 n æ)^{2}} \alpha=K^{(P)}(\lambda) \cdot D .
$$

Relations (4) and (3) imply the following:

$$
\tau(\lambda)=\frac{1.5 C_{m} l}{\rho_{k}} K^{(p)}(\lambda)=C \cdot K^{(p)}(\lambda) \cdot C_{m} .
$$

As it can be seen from formula (5), $\tau(\lambda)$ does not depend on $D$ and, therefore, concentration $C_{m}$ can be determined from relation (1). Moreover, it can be shown that this method of determination of $C_{m}$ can also be used for a polydisperse medium in case if Rayleigh approximation conditions are met for all particles [1]

$$
\tau(\lambda)=\frac{1.5 C_{m} l}{\rho_{k}} \cdot \frac{\int_{0}^{\infty} Q(\alpha, m) f(D) D^{2} d D}{\int_{0}^{\infty} f(D) D^{3} d D} .
$$


where $f(D)$ - particle-size distribution function.

If Rayleigh approximation conditions are met for all particles, then from equations (6) and (4) we have:

$$
\tau(\lambda)=\frac{1.5 C_{m} l}{\rho_{k}} \cdot \frac{K^{(P)}(\lambda) \int_{0}^{\infty} D f(D) D^{2} d D}{\int_{0}^{\infty} f(D) D^{3} d D}=\frac{1.5 C_{m} l}{\rho_{k}} K^{(P)}(\lambda) .
$$

Thus, expression (7) is identical to equation (5).

$C_{m}$ calculation from formula (1) using measured values of optical density $\lambda^{\text {meas }}(\lambda)$ is considered valid only for a limited wavelength range $\lambda \geq \lambda$ *

Wavelength $\lambda_{*}$ boundary value is determined from the following relation

$$
\bar{\tau}(\lambda)=\frac{\tau^{\text {meas }}(\lambda)}{K^{(p)}(\lambda)} .
$$

The graph of function has two sections (fig.1) - monotonic decrease $\left(\lambda<\lambda_{*}\right)$ and constant $\left(\lambda>\lambda_{*}\right)$; the joint of these sections determines the wavelength boundary value $\lambda_{*}$ of the area of Rayleigh approximation. Rayleigh approximation is not performed for area $\lambda<\lambda_{*}$ and calculation using relation (1) gives large error values. Concentration $C_{m}$ for the range $\lambda \geq \lambda_{*}$ is determined using the following formula:

$$
C_{m}=\frac{\bar{\tau}_{*}}{C}=\frac{\rho_{k}}{1.5 l} \cdot \bar{\tau}_{*} .
$$

A value $\alpha_{*}=f(\lambda)$, depending on wavelength $\lambda$ is necessary to calculate maximum particle size $D_{m}$ using expression (2). Illustration of this dependence for soot is given in fig.2. These charts were built on the basis of condition of $5 \%$ and $10 \%$ deviation of $Q(\lambda)$ from $Q(p)(\lambda)$ (fig.3). It should be noted that Rayleigh approximation condition is met for $\alpha$ $<\alpha_{*}$ with specified controlled accuracy.

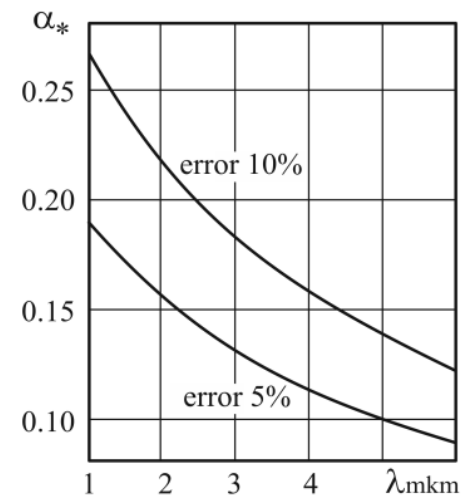

Fig. 2. Dependence of $\alpha_{*}$ on wavelength $\lambda$.

Dependence $\alpha_{*}(\lambda)$ has exponential character and can be approximated by the following equation:

$$
\alpha_{*}=a \cdot \exp (-b \lambda)
$$

Expression for $D_{m}$ can be obtained from relations (8) and (2): 


$$
D_{m}=\frac{\lambda_{*}}{\pi} a \cdot \exp (-b \lambda)
$$

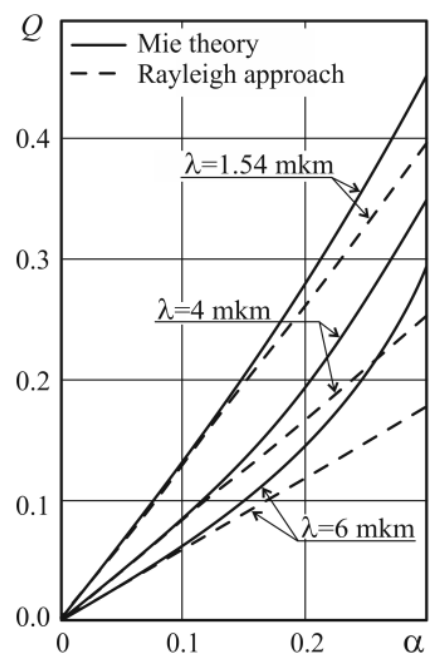

Fig. 3. Dependence of attenuation efficiency factor $Q$ on diffraction parameter $\alpha$.

According to the data from [2] for $\lambda=(1 \div 6) \mu \mathrm{m}$ the dependence of refractive index $\mathrm{n}$ and attenuation coefficient $æ$ on wavelength $\lambda$ is as follows: $n=1.6-0.3 \lambda, \mathfrak{x}_{*}=0.6 \lambda^{0.6}$.

Approximation error in this case comprises $(2.5 \div 3.0) \%$. Final expression for maximum particle size $D_{m}$ for strictly determined dependence $\alpha_{*}=0.217 \cdot \exp (-0.155 \lambda)$ is as follows:

$$
D_{m}=\frac{0.217 \lambda_{*}}{\pi} \cdot \exp (-0.155 \lambda) .
$$

This method makes it possible to determine both particle concentration and maximum particle size with controlled accuracy for various flames without any initial information about particle size distribution.

Soot samples were collected in the process of combustion of the system in a constantpressure de-vice equipped with a quartz collector and an accessory for slow pressure drop preventing loss of solid combustion products. Unrestricted cylindrical propellant samples with the diameter of $10 \mathrm{~mm}$ and height of $70 \mathrm{~mm}$ were studied, their weight comprised $(10 \div 12)$ g. Quartz collector after burning up and a slow pressure drop was placed into a dryer and was dried for $2-3$ hours at $70-75^{\circ} \mathrm{C}$ until a constant weight was reached. Soot content was determined using the following relation:

$$
C=\frac{P_{2}-P_{1}}{P_{\text {samp }}} \cdot 100 \%,
$$

where $P_{1}$ - weight of pure quartz collector, $P_{2}$ - weight of quartz collector with soot after drying,

$P_{\text {samp }}$ - weight of studied sample.

Chemical analysis of soot [3] indicated that along with carbon particles it contains ammonium chloride. The quantity of the latter varies as oxidizer dispersity and sample combustion pressure change. Ammonium chloride content in a number of cases reaches 60 $\%$ of soot weight. Possible chemical interaction between chlorine and carbon and formation of so called insertion compounds is shown in [4]. 
The results obtained for composition of solid combustion products given in Table 1 indicate that increase of size of oxidizer crystals leads to increase of carbon content in solid combustion products and at the same time reduction of ammonium chloride content compared with systems containing oxidizer particles with sizes less that $50 \mu$.

Comparison of the data obtained by optical diagnostic methods and selection of solid combustion products is given in Table 2 . The data obtained by product selection represent only carbon content (w/o ammonium chloride).

The analysis performed has indicated that results obtained using different methods are comparable. Relatively low soot content in combustion products in case of use of laser diagnostics can likely be explained by deposition of particles on inner walls of the device containing burning sample.

Table 1. Soot composition.

\begin{tabular}{|c|c|c|c|c|c|}
\hline \multirow{2}{*}{ AP size } & \multicolumn{5}{|c|}{ carbon $/ \mathrm{NH}_{4} \mathrm{Cl}, \mathrm{wt} \%$, at various pressures } \\
\cline { 2 - 6 } & $1 \mathrm{MPa}$ & $2 \mathrm{MPa}$ & $3 \mathrm{MPa}$ & $6 \mathrm{MPa}$ & $8 \mathrm{MPa}$ \\
\hline Less than $50 \mu$ & $50 / 50$ & $46 / 54$ & $43 / 57$ & $40 / 60$ & $40 / 60$ \\
\hline$(200 \div 300) \mu$ & $67 / 33$ & - & $65 / 35$ & - & $60 / 40$ \\
\hline bidisperse (1:1) & $60 / 40$ & - & $57 / 43$ & - & $50 / 50$ \\
\hline
\end{tabular}

Table 2. Carbon content in combustion products.

\begin{tabular}{|c|c|c|c|c|c|c|}
\hline \multirow{2}{*}{ AP size } & \multirow{2}{*}{ Method } & \multicolumn{5}{|c|}{ Carbon content, wt \%, at various pressures } \\
\cline { 3 - 7 } & & $1 \mathrm{MPa}$ & $2 \mathrm{MPa}$ & $3 \mathrm{MPa}$ & $6 \mathrm{MPa}$ & $8 \mathrm{MPa}$ \\
\hline \multirow{2}{*}{ Less than $50 \mu$} & selection & 3.9 & 3.7 & 2.9 & 1.6 & 1.5 \\
\cline { 2 - 7 } & optical & 3.7 & 3.6 & 2.5 & 1.3 & 1.4 \\
\hline \multirow{2}{*}{$(200 \div 300) \mu$} & selection & 8.8 & 5.2 & 4.1 & 3.3 & 3.1 \\
\cline { 2 - 7 } & optical & 8.5 & 4.8 & 3.5 & - & - \\
\hline \multirow{2}{*}{ bidisperse $(1: 1)$} & selection & 3.3 & 3.3 & 1.7 & 0.8 & 0.7 \\
\cline { 2 - 7 } & optical & 2.9 & 2.9 & 1.4 & 0.8 & 0.8 \\
\hline
\end{tabular}

Thus, dispersity of oxidizer affects both total soot content in combustion products and chemical composition of solid combustion products. It is noted that increase of particle size leads to increase of carbon content in solid combustion products. In case of use of bidisperse oxidizer leads to reduction of soot content.

\section{Acknowledgments}

This research was supported by the Ministry of Education and Science of the Russian Federation under the Federal Target Program "Research and development on priority directions of scientific-technological complex of Russia for 2014-2020", the agreement No. 14.578.21.0034, unique identifier PNI RFMEFI57814X0034.

\section{References}

1. V.A. Arkhipov, S.S. Bondarchuk, Optical diagnostic methods of heterogeneous plasma combustion products: Study guide (Tomsk State University, Tomsk, 2012) [in Russian] 
2. A.G. Blokh, Heat exchange in steam boilers (Energoatomizdat, Leningrad, 1984) [in Russian]

3. V.A. Klimova, Basic methods of analysis of organic compounds (Khimiya, Moscow, 1975) [in Russian]

4. Weygand-Hilgetag, Experimental Methods in Organic Chemistry (John Wiley \& Sons Inc, 1972)

5. V.A. Arkhipov, S.S. Bondarchuk, Formula of parameters communication of unimodal distributions of particles in the sizes with geometrical characteristics of probability density function (Mechanics of fast flow processes. Tomsk: TSU, 1989) [in Russian]

6. V.A. Arkhipov, S.S. Bondarchuk, N.G. Kvesko, A.T. Roslyak, V.F. Trofimov, Athmospheric and Oceanic Optics 17, 5 (2004) 